\title{
A Switched-Capacitor Digital Capacitance Meter
}

HIROKI MATSUMOTO, STUDENT MEMBER, IEEE, AND KENZO WATANABE, MEMBER, IEEE

\begin{abstract}
A digital capacitance meter has been developed based on the switched-capacitor cyclic analog-to-digital (A/D) converter. It consists of the analog arithmetic and sample/hold $(\mathrm{S} / \mathrm{H})$ circuits. The arithmetic circuit first samples the capacitance to be measured in a form of its proportional voltage. This voltage is then converted into a binary number by the $A / D$ converter. The whole operation is insensitive to parasitic capacitances, off set voltages of op amps, and the capacitance mismatch involved in the circuit. Therefore, the proposed meter permits an accurate capacitance measurement. Error analysis shows that 12-bit accuracy can be expected by realizing the meter in an IC form. A prototype meter built using discrete components and examples of measurement are also given.
\end{abstract}

\section{INTRODUCTION}

A CAPACITANCE measurement is indispensable for characterizing $R C$-active and switched-capacitor circuits and capacitive transducers. Conventional methods using Schering, transformer-ratio arm, or four-terminalpair bridge are useful for the measurement of discrete capacitors [1], [2]. They are, however, not applicable to integrated MOS capacitors, because they cannot separate contributions of parasitic capacitances.

The accurate measurement of integrated MOS capacitors is only possible by the on-chip built-in measuring system with digital output. Two such candidates have been reported so far [3], [4], but they are applicable only to floating capacitors. In addition, the chip area required for the implementation is appreciable because of the large device count.

To eliminate these difficulties, a new capacitance meter has been developed based on the switched-capacitor cyclic analog-to-digital (A/D) converter [5]. This paper describes the meter configuration, the principles of measurement, and the expected accuracy when it is realized in an IC form. A prototype meter built using discrete components and examples of measurement are also presented.

\section{Meter Configuration}

Fig. 1 shows the schematic diagram of the digital capacitance meter. It consists of two main parts: the analog arithmetic circuit formed by op-amp $A_{1}$ and two capacitors $C_{1}$ and $C_{2}$, and the sample/hold $(\mathrm{S} / \mathrm{H})$ circuit formed by op-amp $A_{2}$ and capacitor $C_{3}$. The capacitor under measurement (CUM) is connected between the terminals $X_{F}{ }^{-}$ $X_{F}^{\prime}$ if it is floating, or to the terminal $X_{G}$ if it is grounded.

Manuscript received October 7, 1985; revised April 7, 1986.

The authors are with the Research Institute of Electronics, Shizuoka University, Hamamatsu 432, Japan.

IEEE Log Number 8610229.

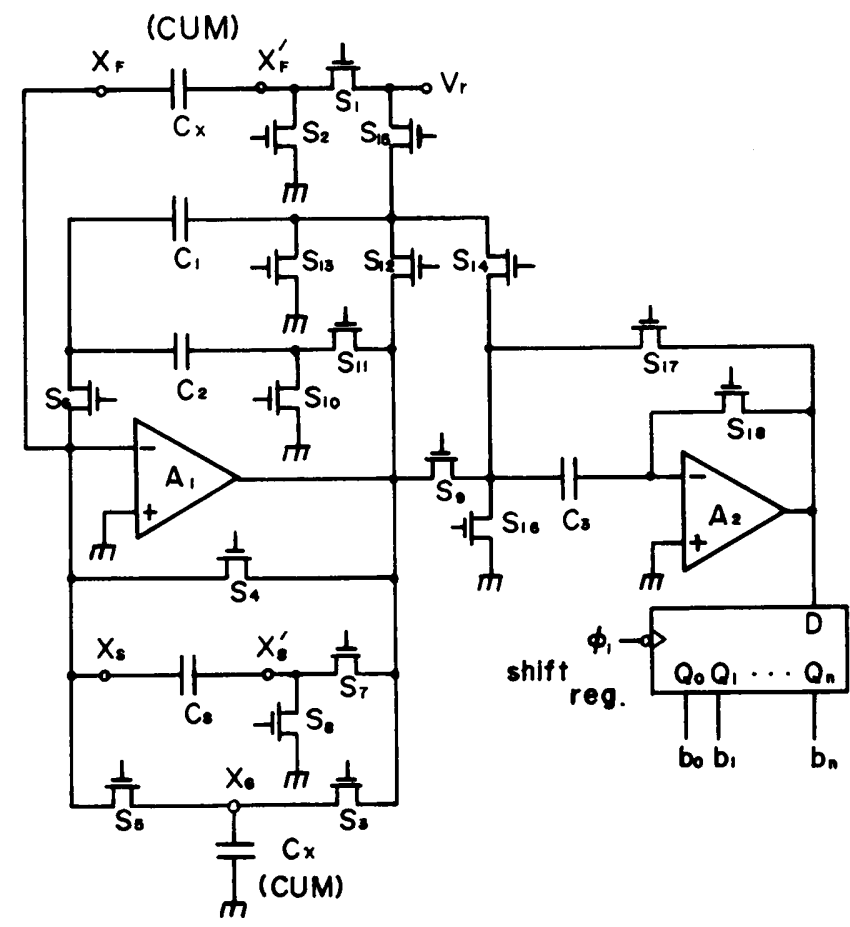

Fig. 1. The schematic diagram of the digital capacitance meter.

Its value is to be measured with reference to the capacitor $C_{S}$ connected between the terminals $X_{S}-X_{S}^{\prime} . V_{r}$ is the reference voltage. It may assume any value, but having its voltage as high as possible is preferable to enhance measurement accuracy, unless this causes saturation of the op amps.

Meter operation is controlled by digital signals, whose timing is shown in Fig. 2. A "reset" signal initializes the meter by closing all the even-numbered switches except $S_{14}$ in Fig. 1. The mode signal distinguishes between the "sample" state in which the arithmetic circuit samples the voltage proportional to the capacitance ratio $C_{X} / C_{S}$ and the "convert" state in which the whole circuit performs the cyclic A/D conversion of the voltage, sampled in the preceding "sample" state, into a binary number. Driving the switches, the nonoverlapping five-phase clock $\phi_{i}(i=1,2, \cdots, 5)$ manages the operation in each state.

\section{A. Operation in the "Sample" State}

The analog arithmetic circuit forms the amplifier in this state. Its configuration depends on which capacitor, a floating or a grounded capacitor, is to be measured.

Fig. 3(a) shows the amplifier configuration for a floating-capacitor measurement. The clock signal which drives each switch is also shown next to its control terminal to identify the following operation in each phase. The switch 


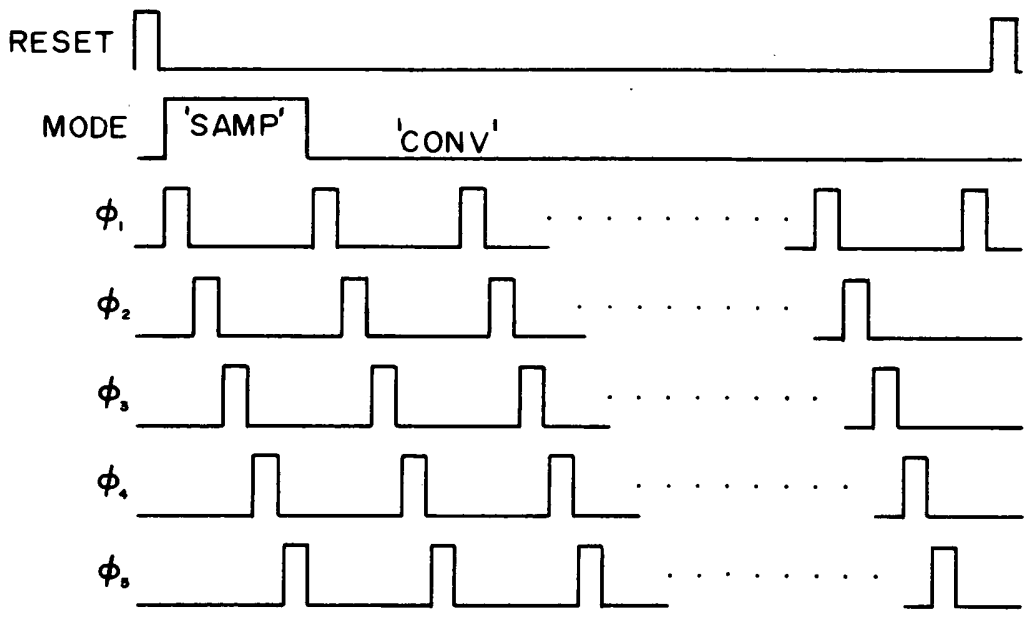

Fig. 2. Timing diagram of digital signals controlling the meter operation.

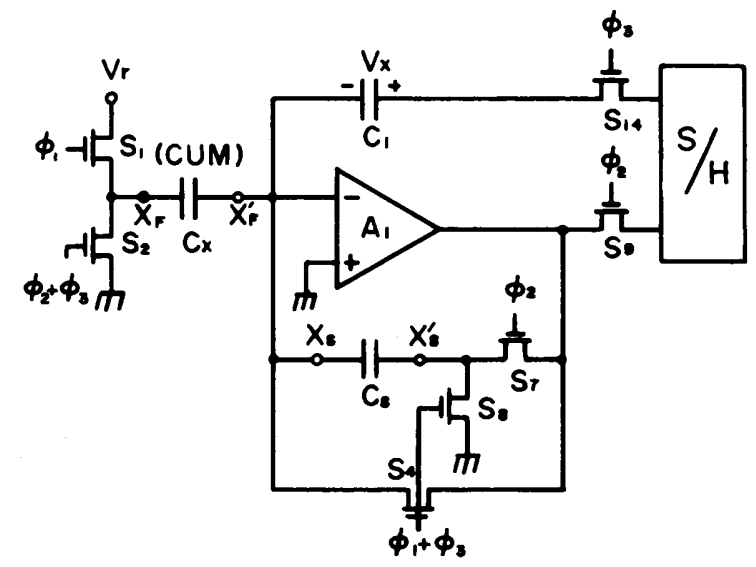

(a)

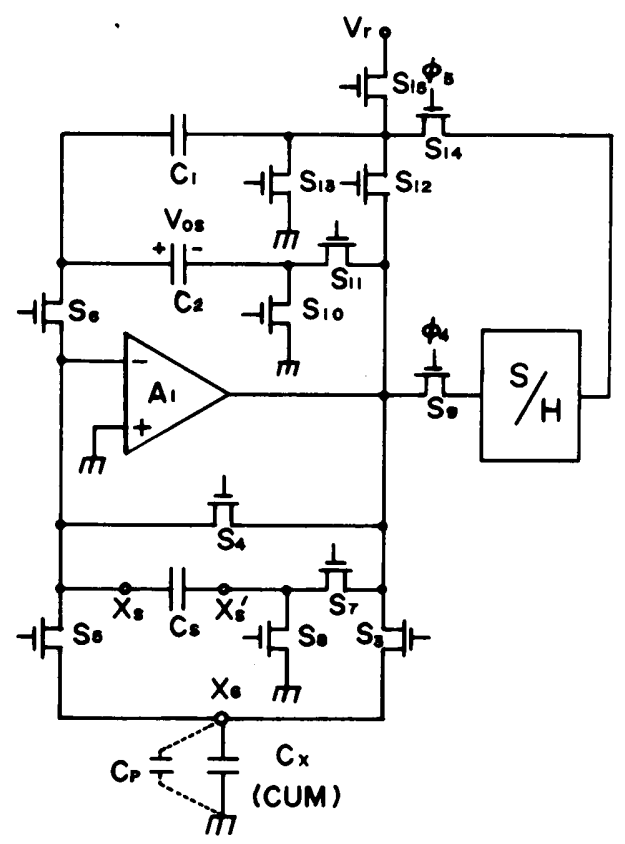

(b)

Fig. 3. The circuit configurations in the "sample" state for (a) floatingand (b) grounded-capacitor measurements.

$S_{6}$ is kept on during this state and hence omitted. In the $\phi_{1}$ phase, $C_{X}$ is charged to the reference voltage $V_{r}$, while $C_{S}$ is discharged to ground. In the next $\phi_{2}$ phase, the charge stored in $C_{X}$ is transferred to $C_{S}$. This produces the voltage $V_{x}$ :

$$
V_{x}=\left(C_{X} / C_{S}\right) V_{r}
$$

TABLE I

SWitching Sequence in the "SAMPLE"' State for Grounded-CaPacitor MEASUREMENT

\begin{tabular}{|c|c|c|c|c|c|c|c|c|c|}
\hline$\phi s$ & $\mathrm{~s}_{3}$ & $\mathrm{~s}_{4}$ & $s_{5}$ & $\mathrm{~s}_{6}$ & $\mathrm{~s}_{7}$ & $\mathrm{~s}_{8}$ & $\mathrm{~s}_{10} \mathrm{~s}_{11}$ & $\mathrm{~s}_{12} \mathrm{~s}_{13}$ & ${ }_{3} s_{15}$ \\
\hline$\phi_{1}$ & & & & 1 & & 1 & 1 & & 1 \\
\hline$\phi_{2}$ & & 1 & & 1 & & 1 & & 1 & \\
\hline$\phi_{3}$ & 1 & & & 1 & & 1 & 1 & 1 & \\
\hline$\phi_{4}$ & & & 1 & & 1 & & 1 & & \\
\hline$\phi_{5}$ & & 1 & & 1 & & 1 & 1 & & \\
\hline
\end{tabular}

This voltage is sampled and held by the $\mathrm{S} / \mathrm{H}$ circuit. In the $\phi_{3}$ phase, the $\mathrm{S} / \mathrm{H}$ circuit charges $C_{1}$ to $V_{x}$ through $S_{14}$ and $S_{4}$, while the arithmetic circuit discharges $C_{2}$ and $C_{S}$.

This completes the operation in the "sample" state. The capacitors $C_{1}$ and $C_{3}$ hold the voltage $V_{x}$ until the subsequent "convert" state. It is noted here that the above operation is insensitive to both the offset voltages of op amps and parasitic capacitances, and thus $V_{x}$ does not include these nonideal components [6], [7].

The amplifier configuration for grounded-capacitor measurement is shown in Fig. 3(b). A somewhat elaborate switching sequence, which is shown in Table $I$, is adopted to assure op-amp $A_{1}$ of the offset-free amplification. The " 1 "' in this table marks those switches that are ON in the corresponding phase. Referring to this table, one can identify the operation in each phase.

In the $\phi_{1}$ phase, the arithmetic circuit forms the inverting amplifier. Its input is the reference voltage $V_{r}$ and its gain is $-\alpha$, where $\alpha=C_{1} / C_{2}$. Since $C_{1}$ was discharged to 0 while $C_{2}$ was charged to the offset voltage $V_{o s}$ of opamp $A_{1}$ with the polarity shown in Fig. 3(b) in the preceding "reset" phase, the voltage across $C_{2}$ becomes $-\alpha\left(V_{r}-V_{o s}\right)$. After discharging $C_{1}$ through $S_{4}$ and $S_{13}$ in the $\phi_{2}$ phase, the arithmetic circuit forms the noninverting amplifier in the $\phi_{3}$ phase. Its input is the voltage stored in $C_{2}$ in the preceding $\phi_{1}$ phase and its gain is $1 / \alpha$. The output voltage of $A_{1}$ is now $-V_{r}+V_{o s}$, which is stored in $C_{X}$ through $S_{3}$. In the $\phi_{4}$ phase, op-amp $A_{1}$ forms the inverting amplifier with $C_{X}$ and $C_{S}$ being the input and feedback capacitors, respectively. The input is the voltage stored in $C_{X}$ in the last $\phi_{3}$ phase. This produces the 


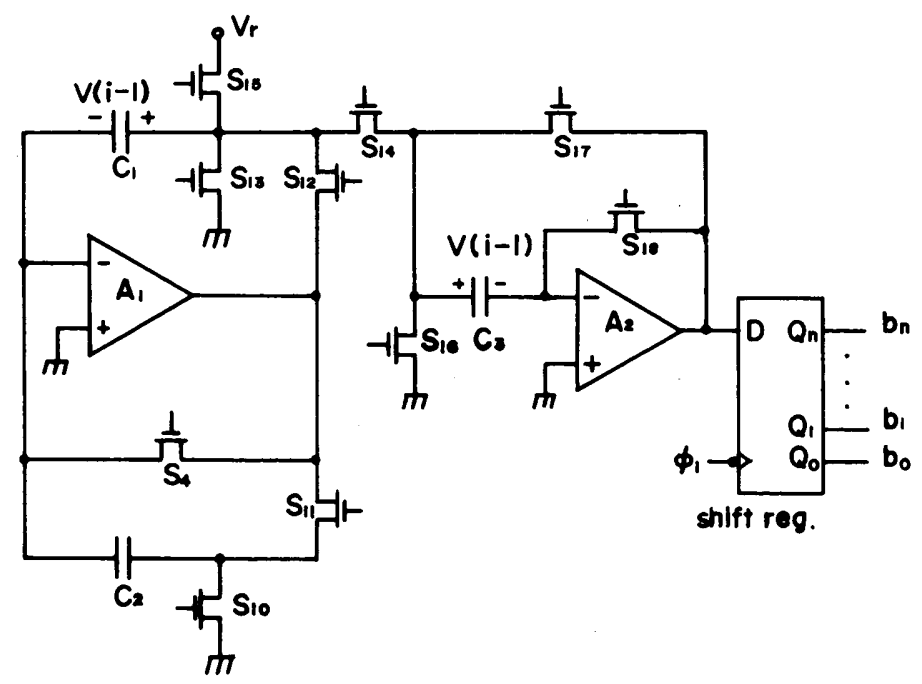

Fig. 4. The circuit configuration in the "convert" state.

output voltage $V_{x}$ given by (1). It is noted here again that $V_{x}$ does not include the offset voltage $V_{o s} . V_{x}$ is also stored in $C_{3}$ through $S_{9}$ and $S_{18}$. Discharging $C_{S}$ through $S_{4}$ and $S_{8}$ and charging $C_{1}$ to $V_{x}$ by the $\mathrm{S} / \mathrm{H}$ circuit in the $\phi_{5}$ phase, the circuit completes the operation in the "sample"' state.

\section{B. Operation in the "Convert"' State}

In this state, the analog arithmetic and $\mathrm{S} / \mathrm{H}$ circuits form the cyclic A/D converter shown in Fig. 4 [5]. It converts the voltage $V_{x}$ produced in the preceding "sample" state into the $n$-bit binary number $b$, according to the following algorithm [8]:

$$
\begin{aligned}
V(i) & =2 V(i-1)+(-1)^{b_{i-1}} V_{r} \\
b_{i} & =\left\{\begin{array}{ll}
1, & \text { if } V(i) \geq 0 \\
0, & \text { otherwise }
\end{array} \quad(i=1,2, \cdots, n)\right.
\end{aligned}
$$

where $V(0)=V_{x}$, and $b_{1}$ and $b_{n}$ are the most-significant and least-significant bits of $b$, respectively. The analog arithmetic circuit executes the operation involved in (2), while, operating also as a comparator, the $\mathrm{S} / \mathrm{H}$ circuit executes the operation involved in (3). Switching sequence for executing the conversion algorithm is listed in Table II.

Let the capacitors $C_{1}$ and $C_{3}$ store the voltage $V(i-1)$, and let $C_{2}$ be discharged to 0 . In the $i$ th cycle of operation, $V(i)$ given by (2) is produced by the following steps. (Refer also to Table II to identify the operation in each step.)

1) $\phi_{1}$ phase: The analog arithmetic circuit forms the noninverting amplifier. Its input is $V(i-1)$ stored in $C_{1}$. The voltage across $C_{2}$ is then $\alpha V(i-1)$. Operating as a comparator, op amp $A_{2}$ tests the polarity of $V(i-1)$ stored in $C_{3}$. If it is positive, then $b_{i-1}$ is set to 1 . Otherwise, it is reset to 0 . The shift register stores the value $b_{i-1}$.

2) $\phi_{2}$ phase: If $b_{i-1}=1$, then the arithmetic circuit forms the inverting integrator to execute the subtraction involved in (2). Its input is $V_{r}$. The voltage across $C_{2}$ thus becomes $\alpha\left\{V(i-1)-V_{r}\right\}$. If $b_{i-1}=0$, on the other
TABLE II

\begin{tabular}{|c|c|c|c|c|c|c|c|}
\hline$\$$ & $\mathrm{~s}_{4}$ & $\mathrm{~s}_{10} \mathrm{~s}_{11} \mathrm{~s}_{1}$ & ${ }_{2} s_{1}$ & ${ }_{13} s_{14}$ & $\mathrm{~s}_{15}$ & $\mathrm{~s}_{16} \mathrm{~s}$ & $\mathrm{~s}_{17} \mathrm{~s}_{18}$ \\
\hline \multirow{5}{*}{$\begin{array}{l}\phi_{1} \\
\phi_{2} \\
\phi_{3} \\
\phi_{4} \\
\phi_{5}\end{array}$} & \multirow{5}{*}{$\begin{array}{c}\bar{b}_{i-1} \\
b_{i-1} \\
1\end{array}$} & 1 & 1 & & & 1 & \\
\hline & & $b_{i-1}$ & & & 1 & & 1 \\
\hline & & $\overline{\mathrm{b}}_{i-1}$ & 1 & & & & 1 \\
\hline & & & & 1 & & & 1 \\
\hline & & 1 & & 1 & & & 1 \\
\hline
\end{tabular}

Switching Sequence in the "Convert" State

hand, the circuit charges $C_{1}$ to $V_{r}$ through $S_{15}$ and $S_{4}$. Opamp $A_{2}$ now operates as a hold circuit.

3) $\phi_{3}$ phase: If $b_{i-1}=0$, the arithmetic circuit transfers the charge stored in $C_{1}$ in the last $\phi_{2}$ phase into $C_{2}$. This executes the addition in (2) and the voltage across $C_{2}$ now becomes $\alpha\left\{V(i-1)+V_{r}\right\}$. Otherwise, $C_{1}$ is discharged to ground through $S_{4}$ and $S_{13}$.

4) $\phi_{4}$ phase: The hold op-amp $A_{2}$ charges $C_{1}$ to $V(i-$ $1)$.

5) $\phi_{5}$ phase: The arithmetic circuit forms the noninverting integrator. Its input is the voltage stored in $C_{2}$ in the previous $\phi_{3}$ phase. The voltage across $C_{1}$ then becomes $V(i)$ given by (2). $C_{3}$ is also charged to $V(i)$ through $S_{14}$ and $S_{18}$.

This completes one cycle of operation. Repeating this conversion cycle $n$ times, the circuit generates the $n$-bit number $b$, which is related to the capacitance $C_{X}$ as

$$
C_{X}=\sum_{i=1}^{n} b_{i} 2^{-i} C_{S}
$$

The conversion process is also insensitive to offset voltages of op amps, parasitic capacitances, and the capacitance ratio $\alpha$.

\section{Accuracy Estimate}

The main error sources in this meter are the feedthrough of digital signals and the finite open-loop gains of op amps. The parasitic capacitance $C_{p}$ between the node $X_{G}$ and ground (Fig. 3(b)) also causes the error in groundedcapacitor measurement.

All the switches shown in Fig. 1 involve the feedthrough problem, but only those switches that are connected to the inverting input terminals of op amps are considered here, because their effect is most serious. Let $Q_{f}$ be the feedthrough charge injected through $S_{4}$ and $S_{18}$ when they turn their state from ON to OFF. The switches $S_{5}$ and $S_{6}$ also inject the charge $Q_{f}$ when grounded-capacitor measurement is concerned. Their contributions are, however, neglected in a first-order approximation, because they are driven by a complementary digital signal and their feedthrough charges tend to cancel each other.

Taking the feedthrough charge $Q_{f}$ (the finite open-loop gains $A$ of op-amps $A_{1}$ and $A_{2}$ ) and the parasitic capacitance $C_{p}$ into account, the output voltage of $A_{1}$ in the "sample" state and the conversion algorithm executed in the "convert" state can be expressed as follows: 


$$
V_{x}^{\prime}=\left(\frac{C_{X}}{C_{S}}\left(\frac{A}{1+A}\right)^{2}+\frac{C_{r e s}}{C_{S}}\right) V_{r}
$$

where $C_{r e s}=C_{p}+Q_{f} / V_{r}$, and

$$
\begin{aligned}
V(i)= & 2\left(\frac{A}{1+A}\right)^{2} V(i-1)+(-1)^{b_{i-1}}\left(\frac{A}{1+A}\right)^{2} V_{r} \\
& +\left(2+\bar{b}_{i-1}\right) \frac{Q_{f}}{C_{1}} \quad(i=1,2, \cdots, n)
\end{aligned}
$$

where $V^{\prime}(0)=V_{x}^{\prime}$, and a prime denotes the erroneous quantity.

Using (5) and (6), one can estimate separately the contribution of each error source; the capacitance meter measures the residual capacitance $C_{r e s}$ when $C_{x}$ is disconnected. Therefore the contribution of $C_{r e s}$ can be eliminated by subtracting it from the measured capacitance $C_{X}^{\prime}$. The finite gain $A$ reduces the gain of the amplifier in the "sample" state. This reduction in gain is equivalent to scaling the reference voltage $V_{r}$ by $(A /(1+A))^{2}$, as indicated in (5). The scaled reference voltage is also included in the second term in the right-hand side of (6). Thus the gain reduction in the "sample" state is canceled by the scaled reference voltage in the cyclic A/D conversion.

Executing the algorithm given by (6) $n$ times, the cyclic A/D converter produces, to a first order, the error voltage

$$
\begin{aligned}
\Delta V= & \Delta V_{A}+\Delta V_{f} \\
= & -2\left\{n \cdot 2^{n} V(0)+\sum_{i=1}^{n}(-1)^{b_{i-1}}(n-i) 2^{n-i} V_{r}\right\} / A \\
& +\left(2^{n+1}+\sum_{i=1}^{n} \bar{b}_{i-1} 2^{n-i}\right) Q_{f} / C_{1}
\end{aligned}
$$

where $\Delta V_{A}$ and $\Delta V_{f}$ are the error voltages caused by the finite open-loop gain $A$ and the feedthrough charge $Q_{f}$, respectively. When $V(0)=V_{r}$, and hence all $b_{i}$ 's assume $1, \Delta V_{A}$ becomes maximum

$$
\left[\Delta V_{A}\right]_{\max }=2^{n+2} V_{r} / A .
$$

For the A/D conversion to be accurate down to its LSB, this error voltage should be smaller than $V_{r}$. Thus we have

$$
2^{n+2}<A .
$$

If $A=80 \mathrm{~dB}$, the conversion accuracy is estimated to be 12 bits.

The error voltage $\Delta V_{f}$ becomes maximum when all $b_{i}$ 's assume 0:

$$
\left[\Delta V_{f}\right]_{\max }=3 \times 2^{n+1} Q_{f} / C_{1}
$$

Thus we can obtain the accuracy condition

$$
3 \times 2^{n+1}<C_{1} V_{r} / Q_{f}
$$

The charge ratio $C_{1} V_{r} / Q_{f}$ as high as $5 \times 10^{4}$ can be realized with presently available MOS technologies by ac-

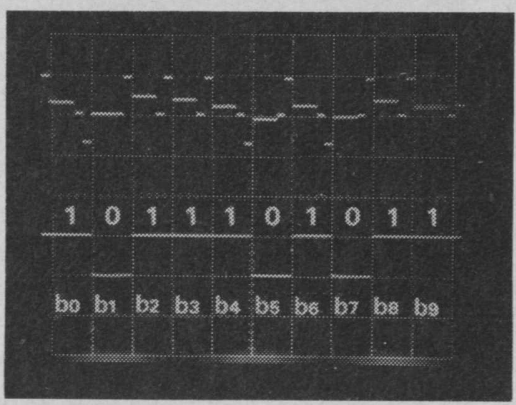

Fig. 5. Experimentally observed waveforms when the meter measured a grounded capacitor of nominal value $470 \mathrm{pF}$ with reference to $1039-\mathrm{pF}$ capacitor. Upper trace: the output voltage waveform of op-amp $A_{2}$. Lower trace: the bit pattern obtained. Horizontal scale: $625 \mu \mathrm{s} / \mathrm{div}$; and vertical scale: $5 \mathrm{~V} /$ div

commodating the clock feedthrough cancellation scheme and making $C_{1}$ as large as practical [9]. The conversion accuracy limited by $Q_{f}$ is then estimated to be 14 bits.

Summarizing the above discussion, we can conclude that the accuracy of the present capacitance meter is limited by the finite open-loop gains of op amps or the feedthrough charge and a 12-bit quantization accuracy can be obtained by fabricating it in an MOS IC form.

\section{Measurement Examples}

A prototype capacitance meter was built using discrete components to confirm the principles of measurement. Op amps and switches used were LF353 and MC14016, respectively. Capacitors $C_{1}, C_{2}$, and $C_{3}$ were chosen to be $2.2 \mathrm{nF}$. The reference voltage $V_{r}$ was set to $2 \mathrm{~V}$. The clock feedthrough cancellation technique was not used.

Fig. 5 shows waveforms observed during the "convert" state when a capacitor of nominal value $470 \mathrm{pF}$ (its actual value measured by the commercial four-terminalpair bridge ${ }^{1}$ was $463 \mathrm{pF}$ ) was subjected to grounded-capacitor measurement using the reference capacitor of 1039 $\mathrm{pF}$. One horizontal division corresponds to one conversion cycle of five phases. The upper trace shows the output voltage of op-amp $A_{2}$. The voltage $V(i)$ held in $C_{3}$ can be seen during the first three phases of each cycle. The output in the last phase of each cycle indicates the polarity of $V(i)$, which is reflected in the bit pattern $b_{0} b_{1} \cdots b_{n}$ $=1011101011$ shown in the lower trace. The first bit $b_{0}$ is the sign bit. The measured capacitance including the residual capacitance $10.1 \mathrm{pF}$, which was measured separately by disconnecting $C_{X}$, was thus $1039 \times(235 / 512)$ $=476.9 \mathrm{pF}$. The large residual capacitance was attributed to clock feedthrough.

A number of capacitors were measured by both the floating and grounded methods. Table III lists the measured results together with those obtained by the abovementioned commercial bridge. The discrepancies between them are mostly within 1 percent. Errors which were caused by clock feedthrough will be greatly reduced by accommodating the clock feedthrough cancellation scheme in its integrated realization.

'HP Model 4275A multifrequency LCR meter. 
TABLE III

Comparison Between the Capacitances $C_{\text {DCm }}$ Measured by the Present Digital Capacitance Meter and those $C_{\text {ftb }}$ By the Conventional Four-Terminal-Pair Bridge

The capacitances in parentheses were obtained by the grounded measurement. $C_{\text {nom }}$ represents the nominal value and $\epsilon_{r}$ is the relative deviation between $C_{\mathrm{DCM}}$ and $C_{\mathrm{FTB}}$.

\begin{tabular}{|c|c|c|c|c|c|c|}
\hline$C_{\text {nom }} p F$ & \multicolumn{3}{|c|}{$\mathrm{C}_{\mathrm{DCM}} \quad \mathrm{pF}$} & $\mathrm{C}_{\text {FTB }} \mathrm{pF}$ & ${ }^{\varepsilon}{ }_{r}$ & \\
\hline 1 & 0.914 & 0.885 & & 0.9 & 1.5 & $(1.5)$ \\
\hline 2 & 1.91 & 1.892 & & 1.9 & 1.0 & $(0.8)$ \\
\hline 4 & 3.79 & 3.81 & ) & 3.8 & 1.0 & $(1.0)$ \\
\hline 10 & 10.22 & 10.21 & ) & 10.3 & 0.78 & $(0.87)$ \\
\hline 20 & 20.37 & 20.35 & ) & 20.2 & 0.84 & $(0.74)$ \\
\hline 47 & 47.78 & 47.10 & ) & 47.4 & 0.8 & $(0.63)$ \\
\hline 100 & 97.58 & 99.13 & ) & 98.2 & 0.63 & $(0.95)$ \\
\hline 220 & 234.5 & 233.9 & ) & 236 & 0.64 & $(0.90)$ \\
\hline 470 & 459.0 & 466.8 & ) & 463 & 0.86 & $(0.82)$ \\
\hline 1000 & 1075 & 1073 & ) & 1065 & 0.94 & $(0.78)$ \\
\hline 2200 & 2870 & 2917 & ) & 2890 & 0.69 & $(0.93)$ \\
\hline 4700 & 4730 & 4731 & ) & 4700 & 0.64 & $(0.66)$ \\
\hline 10000 & 14270 & ( 14261 & ) & 14350 & 0.8 & $(0.89)$ \\
\hline 22000 & 21910 & ( 21903 & ) & 21700 & 0.97 & $(0.94)$ \\
\hline
\end{tabular}

\section{Conclusions}

A digital capacitance meter using switched-capacitor circuitry has been described. It features high accuracy made possible by its offset-tolerant and parasitic-insensitive circuit configuration. Error analysis has shown that 12-bit accuracy can be expected by fabricating the meter in an IC form using present MOS technologies. The chip area required for the fabrication is very small because of the small device count involved. Therefore it would be useful as an on-chip meter to characterize integrated MOS capacitors and also for built-in interfacing of intelligent capacitive transducers.

\section{ACKNOWLEDGMENT}

The authors thank Prof. G. C. Temes of UCLA for useful discussions.

\section{REFERENCES}

[1] B. M. Oliver and J. M. Cage, Electronic Measurement and Instrumentation. New York: McGraw-Hill, 1971, ch. 9.

[2] A. C. Corney, "A universal four-terminal-pair impedance bridge," IEEE Trans. Instrum. Meas., vol. IM-28, no. 3, pp. 211-215, Sept. 1979.

[3] J. L. McCreary and D. A. Sealer, "Precision capacitor ratio measurement technique for integrated circuit capacitor array," IEEE Trans. Instrum. Meas., vol. IM-28, no. 1, pp. 11-17, Mar. 1979.

[4] K. Watanabe and G. C. Temes, "A switched-capacitor digital capacitance bridge," IEEE Trans. Instrum. Meas., vol. IM-33, no. 4, pp. 247-251, Dec. 1984.

[5] H. Matsumoto and K. Watanabe, "Improved switched-capacitor algorithmic analogue-to-digital converter," Electron. Lett., vol. 21, pp. 430-431, May 1985.

[6] R. Gregorian and S. Fan, "Offset free high-resolution D/A converter," in Proc. 14th Asilomar Conf. Circuits, Systems, and Components, Nov. 1980, pp. 316-319.

[7] K. Martin, "Improved circuits for the realization of switched-capacitor filters," IEEE Trans. Circuits Syst., vol. CAS-27, pp. 237-244, Apr. 1980.

[8] R. H. McCharles and D. A. Hodges, "Charge circuits for analog LSI," IEEE Trans. Circuits Syst., vol. CAS-25, pp. 490-497, July 1978.

[9] K. Martin, "New clock feedthrough cancellation technique for analog MOS switched-capacitor circuits," Electron. Lett., vol. 18, pp. 3940, Jan. 1982. 